\title{
Predatory journal publishing: Is this an alternate universe?
}

The views expressed in this editorial are those of the author and do not necessarily reflect the position of the Canadian Medical Association or its subsidiaries.

$\mathrm{T}$ here has been a proliferation of publications, both peer-reviewed and invited. This has predominantly occurred via an increased number of online journals. In particular, there has been a staggering increase in both the number of publishers and individual publications. One would intuitively think that this has been driven by a heightened demand for space from an increasing number of researchers performing high-quality work, expansion of research techniques and unique methodology that necessitates more niche journals.

The truth is that there is a significant economic driver behind the increase in journal numbers. Profit margin in academic publishing is a badly kept secret. The largest academic publishing firms possess an economic margin in line with some of the richest companies in the world. ${ }^{1}$ This has undoubtedly attracted many new publications into the marketplace, often with low operating costs facilitated by a lack of formal editing, peer review, or professional online depositories. This landscape has resulted in journals now numbering in the thousands within the biomedical space. The sheer volume of journals should ensure that all meaningful research eventually finds an appropriate platform. More specifically, it would be intuitive to think that authors would be able to publish more easily and that readers could access these high-quality publications without firewall obstacles.

Unfortunately, this utopia is far from the reality. Many new journals possess little desire to exact any meaningful oversight of their published content. These journals are typically referred to as "predatory." Not surprisingly, these publications have several unique business plans aimed at profitability. The most obvious of these involves charging significant editorial fees while returning no relevant reviewing, editing, or content adjudication. Reports of published manuscripts with repeated nonsensical phrases or even randomly generated words are increasingly common. Other cases of withholding submissions until supplementary fees are paid, or charging high fees for supposed plagiarism cases, have also been described. ${ }^{2}$ Good quality research can be held up for years in supposed "review" until these demands are met. Some journals have also hijacked otherwise legitimate journal websites via copycat formatting and therefore fool prospective authors into a steady supply of submitted papers for which they can be charged.

Editorial board recruitment of junior academic staff, mixed with additional faculty from high-profile universi- ties, can offer the appearance of respectability. Our mailboxes are unfortunately crammed with invitations to either publish or become editors in journals that only occasionally overlap with our areas of specialty. It is becoming more difficult to separate legitimate from predatory journals, especially as these new journals move through their first few years of publication. It is also no longer as simple as calling out journals that are "open access" given that many reputable journals now publish under Open Access models. Our trainees in particular, suffer from an inability to select the appropriate submission targets.

In the interest of combatting nefarious publications, there are now several lists of both good and bad journals (e.g., Beall's list of potential predatory journals and publishers, Cabell's whitelist, Cabell's journal blacklist). While debate remains regarding a small number of journals, these publicly available listings are reasonable guides. They also offer a good source of which journal articles should carry significant weight in guiding medical decisions. It is critical to remember that there is no current substitute for established journals with a long publishing-house-affiliated history. New journals that arrive on the scene can be important sources of information, particularly in subspecialty areas. Unfortunately, like "alternative facts," alternative journals are more accurately described as both dishonest and predatory.

Edward Harvey, MD; Chad G. Ball, MD, MSc

Coeditors, Canadian Fournal of Surgery

Competing interests: E.J. Harvey is the cofounder and head of medical innovation of NXTSens Inc.; the cofounder and chief medical officer of MY01 Inc., and Sensia Diagnostics Inc.; and the cofounder and director of Strathera Inc. He receives institutional support from J \& J DePuy Synthes, Stryker, MY01, and Zimmer. No other competing interests were declared.

Content licence: This is an Open Access article distributed in accordance with the terms of the Creative Commons Attribution (CC BY-NC-ND 4.0) licence, which permits use, distribution and reproduction in any medium, provided that the original publication is properly cited, the use is noncommercial (i.e., research or educational use), and no modifications or adaptations are made. See: https://creativecommons.org/licenses/by-nc-nd/4.0/

DOI: $10.1503 /$ cjs.009821

\section{References}

1. Time to break academic publishing's stranglehold on research. NewScientist 2018, Nov. 21. Available: https://www.newscientist. com/article/mg24032052-900-time-to-break-academic-publishings -stranglehold-on-research/\#ixzz6wX2PICff (accessed 2021 May 31).

2. Grudniewicz A, Moher D, Cobey KD, et al. Predatory journals: no definition, no defence. Nature 2019;576:210-2. 